\title{
Fibre Bundles and Supergravity
}

\author{
Moustafa A. Awada \\ Theoretical Physics Group, Imperial College of Science and Technology, London SW72BZ, England
}

\begin{abstract}
We present a simple derivation of on-shell $N=2$ supergravity by using fibre bundle analysis; this is done by introducing a central charge as a part of the connection in a principal bundle whose structure group is the superPoincaré group. As a consequence there is a non-trivial generalization of the supersymmetry transformations.
\end{abstract}

\section{Introduction}

Recently [2] it has been proved that $N=1$ supergravity can be constructed in a purely geometrical way by using fibre bundle analysis. This is done by extending the orthonormal frame bundle of a manifold admitting a spin structure to a bundle with structure group, the super-Poincaré group [1].

This method shows its virtues as far as geometrical formulation of supergravity theories are concerned by overcoming certain difficulties [1] that arise when we use a superspace approach; additionally it gives an easy technique for constructing supergravity and its invariances in a more direct way than the work of $[3,4]$.

In this paper we will show how fibre bundle techniques enable us to include matter fields, and in particular to exhibit in a purely geometrical way, a very natural construction of $N=2$ supergravity with its set of supersymmetry transformations. This is done by introducing a central charge as a part of the connection and which has a trivial action on the supersymmetric transformations. This is a natural extension of the work of Yates [1].

\section{Formalism}

Analogously to the $N=1$ supergravity theory discussed in [1], we construct a principal bundle $(E, \pi, M)$ which has structure group, the $N=2$ super-Poincaré group, with a single central charge $Z$. Thus a connection $\Gamma$ in this bundle may be expressed as

$\Gamma=\frac{1}{2} \omega^{a b} J_{a b}+\theta^{a} P_{a}+\psi^{\alpha i} Q_{\alpha i}+A Z$, 
where $J_{a b}$ generates the $\operatorname{SL}(2, C)$ subgroup and $P_{a}$ (respectively, $Q_{\alpha i}$ ) are the generators of boson (respectively, fermionic) translations and $Z$ is the central charge generator of $\mathrm{U}(1)$; note that $\theta^{a}$ (respectively, $\psi^{\alpha i}$ ) is a one-form on $E$ taking values in the even (respectively, odd) part of a Grassmann algebra, and $A$ is a 1 -form on $E(i=1,2)$.

The algebra of the generators in $(2.1)$ is the extended one defined by:

$$
\begin{aligned}
{\left[J_{a b}, J_{c d}\right] } & =\eta_{b c} J_{a d}+\eta_{a d} J_{b c}-\eta_{a c} J_{b d}-\eta_{b d} J_{a c}, \\
\left\{Q_{\alpha i}, Q_{\beta j}\right\} & =\left(C \gamma^{a}\right)_{\alpha \beta} \delta_{i j} P_{a}+C_{\alpha \beta} \varepsilon_{i j} Z, \\
{\left[J_{a b}, Q_{\alpha i}\right] } & =\left(\sigma_{a b}\right)_{\alpha i}^{\beta j} Q_{\beta j} \#_{a b}=\frac{1}{4}\left[\gamma_{a}, \gamma_{b}\right], \\
{\left[J_{a b}, P_{c}\right] } & =\eta_{b c} P_{a}-\eta_{a c} P_{b},
\end{aligned}
$$

where $C_{\alpha \beta}$ is the antisymmetric charge conjugate matrix, and the central charge commutes with any of these generators. This charge is the key ingredient in our construction and makes it significantly different from that in $[3,4]$.

Let $\sigma$ be a cross-section ${ }^{1}$ from $M$ to $E$; then the pull back of $\Gamma$ will give

$$
\begin{aligned}
\sigma^{*} \omega^{a b} & =\omega_{\mu}^{a b} d x^{\mu}, \\
\sigma^{*} \theta^{a} & =e_{\mu}^{a} d x^{\mu}, \\
\sigma^{*} \psi^{\alpha i} & =\psi_{\mu}^{\alpha i} d x^{\mu}, \\
\sigma^{*} A & =A_{\mu} d x^{\mu},
\end{aligned}
$$

where $\omega_{\mu}^{a b}$ is the usual Levi-Civita connection, $e_{\mu}^{a}$ is the vierbein, $\psi_{\mu}^{\alpha i}$ is the usual spin $3 / 2$ field and $A_{\mu}$ is the electromagnetic field. The curvature two-form $\Delta$ is given by the structure equation

$$
\Delta=d \Gamma+\frac{1}{2}[\Gamma, \Gamma]
$$

whence writing

$$
\Delta=\frac{1}{2} \Omega_{E}^{a b} J_{a b}+T^{a} P_{a}+\varrho^{\alpha i} Q_{\alpha i}+H Z
$$

we find

$$
\begin{gathered}
\Omega_{E}^{a b}=d \omega^{a b}+\omega^{a c} \wedge \omega_{c}^{b}, \\
T^{a}=d \theta^{a}+\omega^{a c} \wedge \theta_{c}+\frac{1}{2} \psi^{i} \wedge C \gamma^{a} \psi_{i}, \\
\varrho^{\alpha i}=d \psi^{\alpha i}+\frac{1}{2} \omega^{b c} \wedge\left(\sigma_{b c} \psi\right)^{\alpha i}, \\
H=d A+\frac{1}{2} \psi^{i} \wedge C \psi^{j} \varepsilon_{i j},
\end{gathered}
$$

whose pull back is given by:

$$
\begin{aligned}
\sigma^{*} \Omega_{E}^{a b} & =\frac{1}{2} R_{\mu \nu \mid E}^{a b} d x^{\mu} \wedge d x^{\nu}, \\
\sigma^{*} T^{a} & =\frac{1}{2} T_{\mu \nu}^{a} d x^{\mu} \wedge d x^{\nu} \\
\sigma^{*} \varrho^{\alpha i} & =\frac{1}{2} \varrho_{\mu \nu}^{\alpha i} d x^{\mu} \wedge d x^{\nu} \\
\sigma^{*} H & =\frac{1}{2} F_{\mu \nu} d x^{\mu} \wedge d x^{\nu},
\end{aligned}
$$

1 If $E$ is non-trivial, then local sections must be used 
where

$$
\begin{gathered}
R_{\mu \nu}^{a b}=\partial_{\mu} \omega_{v}^{a b}-\partial_{\nu} \omega_{\mu}^{a b}+\omega_{\mu}^{a c} \omega_{c v}^{b}-\omega_{v}^{a c} \omega_{c \mu}^{b}, \\
T_{\mu \nu}^{a}=\partial_{\mu} e_{\nu}^{a}-\partial_{\nu} e_{\mu}^{a}+\omega_{\mu c}^{a} e_{\nu}^{c}-\omega_{v c}^{a} e_{\mu}^{c}+\psi_{\mu}^{i} C \gamma^{a} \psi_{v i}, \\
\varrho_{\mu \nu}^{\alpha i}=\partial_{\mu} \psi_{\nu}^{\alpha i}-\partial_{\nu} \psi_{\mu}^{\alpha i}+\frac{1}{2} \omega_{\mu}^{e f}\left(\sigma_{e f} \psi_{\nu}\right)^{\alpha i}-\frac{1}{2} \omega_{v}^{e f}\left(\sigma_{e f} \psi_{\mu}\right)^{\alpha i}, \\
F_{\mu \nu}=e_{\mu}^{a} e_{\nu}^{b} F_{a b}=\partial_{\mu} A_{\nu}-\partial_{\nu} A_{\mu}+\psi_{[\mu}^{i} C \psi_{v]}^{j} \varepsilon_{i j},
\end{gathered}
$$

where $e_{\mu}^{a}$ is the inverse of $e_{a}^{\mu}$ such that $e_{\mu}^{a} e_{c}^{\mu}=\delta_{c}^{a}$.

Finally the Bianchi identities read

$$
D \triangle=0,
$$

where $D$ is the horizontal exterior derivative; we deduce

$$
\begin{gathered}
d \Omega_{E}^{a b}+\omega_{c}^{a} \wedge \Omega_{E}^{c b}-\Omega_{E}^{a c} \wedge \omega_{c}^{b}=0, \\
d T^{a}+\omega^{a c} \wedge T_{c}-\Omega_{E}^{a c} \wedge \theta_{c}+\psi^{i} \wedge C \gamma^{a} \varrho_{i}=0, \\
d \varrho^{\alpha i}+\frac{1}{2} \omega^{b c} \wedge\left(\sigma_{b c} \varrho\right)^{\alpha i}-\frac{1}{2} \Omega_{E}^{b c} \wedge\left(\sigma_{b c} \psi\right)^{\alpha i}=0, \\
d H+\psi^{i} \wedge C \varrho^{j} \varepsilon_{i j}=0 .
\end{gathered}
$$

Let us consider the U(1) subgroup of the structure group of $E$ as a theory of Kaluza-Klein type, i.e. let $\pi: \mathrm{U}(1) \times M=P \rightarrow M$ (i.e. $P$ is a sub-bundle of $E$ ), and let $k$ be a metric defined on the real numbers [Lie algebra of $U(1)=L(U(1))]$. Then we can construct a metric $h(g, k, A)$ on $P$, where $g$ is the metric on $M$. Given $a \in \mathrm{U}(1)$, let $R_{a}: P \rightarrow P$ be the corresponding isometry of $E$; then the usual Kaluza-Klein metric $h$ is defined by:

$$
h=\pi^{*} g+k A,
$$

where for $X, Y \in T_{p} P$

$$
\pi^{*} g(X, Y)=g\left(\pi_{*} X, \pi_{*} Y\right) \quad \text { and } \quad k A(X, Y)=k(A(X), A(Y)),
$$

and under the action of $R_{a}$ we have:

$$
h\left(R_{a *} X, R_{a *} Y\right)=h(X, Y) .
$$

Let $\bar{E}_{1}, \bar{E}_{2}, \ldots, \bar{E}_{4}$ be four vector fields on $(M, g)$ defined on a neighbourhood $U$ of $x=\pi(p) ; p \in P$ with their horizontal lifts $E_{1} \ldots E_{4}$ relative to the connection $A$ defined on $\pi^{-1}(U) \in P$. Let $E_{5}$ be a basis of $L(\mathrm{U}(1))$ relative to $k$, then relative to the metric $h$ on $P, E_{1}, \ldots, E_{5}$ will be an orthonormal basis defined on $\pi^{-1}(U)$. With $a, b, c=1, \ldots, 4$ we write

$$
g_{a b}=g\left(\bar{E}_{a}, \bar{E}_{b}\right)=h\left(E_{a}, E_{b}\right)=\eta_{a b}=\operatorname{diag}(\ldots,+), \quad k_{55}=h\left(E_{5}, E_{5}\right)=-1 .
$$

Then following [8], one can prove the following:

$$
R_{555}^{5}=R_{5 a 5}^{5}=R_{55 a}^{5}=R_{a 55}^{5}=0,
$$

also

$$
\begin{aligned}
R_{5 a b}^{5} & =-\left(F_{c a}^{5} F_{5 b}^{c}-F_{c b}^{5} F_{5 a}^{c}\right), \\
R_{a b c}^{5} & =\left(F_{a c, b}^{5}-F_{a b, c}^{5}\right), \\
R_{a 5 b}^{5} & =F_{a b, 5}^{5}+F_{c b}^{5} F_{5 a}^{c},
\end{aligned}
$$


where $R_{B C D}^{A}(A, B=1, \ldots, 5)$ is the Riemannian tensor on $P$ computed from the metric $h$ and expressed in the frame basis $E_{1}, \ldots, E_{5}$. The Ricci tensor is

$$
R_{a b}^{h}=R_{a b}^{g}-2 F_{5 a}^{c} F_{c b}^{5}, \quad R_{a 5}=-F_{5 a, b}^{b}, \quad R_{55}=F_{5 a}^{b} F_{5 b}^{a}
$$

and the scalar curvature is

$$
R_{A}^{A}=R_{a}^{a}+R_{5}^{5}=R_{4-} F_{a b} F^{a b},
$$

where $F_{a b}$ is a matter field strength which includes the electromagnetic field strength, and it is defined through Eqs. (2.7d) and (2.8d) as the U(1) part of the curvature 2-form in Eqs. (2.5) and (2.6d). Now by definition the Riemannian tensor $R^{a b}{ }_{c d}$ is related to the curvature 2 -form in Eq. (2.6a) by

$$
\Omega_{E}^{a b}=\frac{1}{2} R^{a b}{ }_{c d E} \theta^{c} \wedge \theta^{d} .
$$

Therefore, after some manipulations, Eq. (2.13) can be written in 4-form notation as

$$
\Omega_{E}^{a b} \wedge \theta^{c} \wedge \theta^{d} \varepsilon_{a b c d}=\left(\Omega_{4}^{a b}-\frac{1}{3} f^{m m} f_{m m} \theta^{a} \wedge \theta^{b}\right) \wedge \theta^{c} \wedge \theta^{d} \varepsilon_{a b c d} .
$$

Hence in what follows it will be understood that

$$
d \omega^{a b}+\omega^{a c} \wedge \omega^{c b}=\Omega_{E}^{a b}=\Omega_{4}^{a b}-\frac{1}{3} f_{m m} f^{m m} \theta^{a} \wedge \theta^{b},
$$

where $f_{m m}$ is a 0 -form matter field which is antisymmetric tensor representation of $\mathrm{SO}(1,3)$ and is given in the $E_{1}, \ldots, E_{5}$ basis by

$$
f_{m m}=\frac{1}{2} F_{m m},
$$

where $F_{m m}$ is as defined in Eq. (2.13) and (2.8d).

\section{The Lagrangian}

The construction of the lagrangian is very simple provided we bear several facts in mind. In general relativity one can view the Einstein lagrangian as arising from the requirement that the variation of it with respect to the connection $\omega_{\mu}$ should lead to an equation stating that the torsion is zero. Specifically with $L$ being a 4 -form, then

$$
\delta L_{1 \mid \theta=\mathrm{const}}=2 \delta \omega^{c d} \wedge T^{a} \wedge \theta^{b} \varepsilon_{a b c d},
$$

using (2.6a) and (2.6b) with $f_{m m}=0, \psi=0, A=0$ and the fact $D \Omega_{E}^{a b}=0$ ( $D$ is the horizontal exterior derivative $\left.D^{\omega}=d+[\omega],\right)$, one can integrate (3.1) to get

$$
L_{1}=\theta^{a} \wedge \theta^{b} \wedge \Omega_{E}^{c d} \varepsilon_{a b c d},
$$

whose pull back is

$$
\mathscr{L}_{1}=e R,
$$

where $R$ is the scalar curvature and $e=\operatorname{det} e_{\mu}^{a}$. To obtain $N=1$ supergravity we need to drop the condition $\psi=0[1]$; however to get $N=2$ supergravity we need to drop $A=0$, and $f_{m m}=0$, and assume that $\delta L_{N=2}$ will give non-zero torsion. This 
torsion is proportional to $A, F$, and $\theta$. Therefore we expect $\delta L_{N=2}$ to contain at least a part which arises from the variation of the matter field, i.e. $f_{m m}$. The most general expression is

$$
\delta L_{2 \mid \theta, A=\mathrm{const}}=\delta f^{c d} \wedge M^{a} \wedge \theta^{b} \varepsilon_{a b c d},
$$

where $M^{a}$ is a 3 -form which is determined from $T^{a}, \theta^{a}, H$, and $A$. The correct expression is:

$$
M^{a}=\alpha H \wedge \theta^{a}+\beta A \wedge T^{a},
$$

where $\alpha$ and $\beta$ are real coupling constants.

The integration of Eq. (3.1) will give, after using Eqs. (2.6a), (2.6b) and requiring $\operatorname{SL}(2, C)$ invariance:

$$
L_{1}=\theta^{a} \wedge \theta^{b} \wedge \Omega_{E}^{c d} \varepsilon_{a b c d}-4 \psi^{i} \wedge C \gamma_{5} \gamma_{a} \varrho_{i} \wedge \theta^{a}
$$

and the integration of (3.4), using Eq. (3.5) gives

$$
\begin{aligned}
L_{2}= & -\alpha D f^{c d} \wedge \theta^{a} \wedge \theta^{b} \wedge A \varepsilon_{a b c d}+\alpha f^{c d} \psi^{i} \wedge C \gamma^{a} \psi_{i} \wedge \theta^{b} \wedge A \varepsilon_{a b c d} \\
& +\frac{\alpha}{2} f^{c d} \psi^{i} \wedge C \psi^{j} \wedge \theta^{a} \wedge \theta^{b} \varepsilon_{i j} \varepsilon_{a b c d}+K
\end{aligned}
$$

with

$$
K=-(2 \alpha+\beta) \int_{A, \theta=\mathrm{const}} \delta f^{c d} \wedge T^{a} \wedge \theta^{b} \wedge A \varepsilon_{a b c d} .
$$

The lagrangian $L_{2}$ is not invariant under a gauge transformation induced by an element of $\operatorname{SL}(2, C)[1]$, but we make it so by adding the term

$$
-\frac{\alpha}{4}\left(4 d A+\psi^{i} \wedge C \psi^{j} \varepsilon_{i j}\right) \wedge \psi^{k} \wedge C \gamma_{5} \psi^{\ell} \varepsilon_{k \ell},
$$

which will not affect (3.4), and setting $\beta=-2 \alpha$. Then the full lagrangian after using Eq. (2.16) is

$$
\begin{aligned}
L_{N=2}= & \theta^{a} \wedge \theta^{b} \wedge \Omega_{4}^{c d} \varepsilon_{a b c d}-\frac{1}{3} f_{m n} f^{m n} \theta^{a} \wedge \theta^{b} \wedge \theta^{c} \wedge \theta^{d} \varepsilon_{a b c d} \\
& -4 \psi^{i} \wedge C \gamma_{5} \gamma_{b} \varrho_{i} \wedge \theta^{b}-\frac{\alpha}{4}\left(4 d A+\psi^{i} \wedge C \psi^{j} \varepsilon_{i j}\right) \wedge \psi^{k} \wedge C \gamma_{5} \psi^{\ell} \varepsilon_{k \ell} \\
& -\alpha D f^{c d} \wedge \theta^{a} \wedge \theta^{b} \wedge A \varepsilon_{a b c d}+\alpha f^{c d} \wedge \psi^{i} \wedge C \gamma^{a} \psi_{i} \wedge \theta^{b} \wedge A \varepsilon_{a b c d} \\
& +\frac{\alpha}{2} f^{c d} \psi^{i} \wedge C \psi^{j} \wedge \theta^{a} \wedge \theta^{b} \varepsilon_{i j} \varepsilon_{a b c d} .
\end{aligned}
$$

(The invariance of this lagrangian under supersymmetry transformations will be proved in the next section.) Equation (3.8) is the result obtained in $[5,3]$ (after redefining the $\psi$ in (3.8) $\rightarrow i \psi$ and dropping the $i$ (complex number) in the result of [3]).

The above result can be generalized by extending the group of the bundle from the $N=2$ super-Poincaré group to the Orthosymplectic group OSP(4/2). In this case the generators will satisfy 


$$
\begin{gathered}
{\left[J_{a b}, J_{c d}\right]=\eta_{b c} J_{a d}+\ldots,} \\
{\left[P_{a}, P_{b}\right]=J_{a b},} \\
{\left[P_{a}, Q_{\alpha i}\right]=\frac{1}{2}\left(\gamma_{a}\right)_{\alpha i}^{\beta j} Q_{\beta j},} \\
{\left[J_{a b}, Q_{\alpha i}\right]=\left(\sigma_{a b}\right)_{\alpha i}^{\beta j} Q_{\beta j},} \\
\left\{Q_{\alpha i}, Q_{\beta j}\right\}=\left(\sigma^{a b} C\right)_{\alpha \beta} \delta_{i j} J_{a b}+C_{\alpha \beta} \varepsilon_{i j} Z+\delta_{i j}\left(C \gamma^{a}\right)_{\alpha \beta} P_{a}, \\
{\left[J_{a b}, P_{c}\right]=\eta_{b c} P_{a}-\eta_{a c} P_{b},} \\
{\left[Q_{\alpha i}, Z\right]=\varepsilon_{i j} Q_{\alpha j},}
\end{gathered}
$$

with $Z$ commuting with the rest of the generators. The algebra (3.9) closes and the algebra of Eqs. (2.2) may be recovered from (3.9) by rescaling $J_{a b}$, and $Q_{\alpha i}$ (3.9a)-(3.9d) to $m \cdot J_{a b}, m \cdot Q_{\alpha i}$ and taking the limit $m \rightarrow 0$, i.e., a form of WignerInönü contraction. Therefore the contracted $\operatorname{OSP}(4 / 2)$ corresponds to the usual extended super-Poincaré group. Now by using (2.1), (2.4), and (3.9) we obtain the following:

$$
\Delta=\frac{1}{2} \Omega_{E}^{a b} J_{a b}+T^{a} P_{a}+\varrho^{\alpha i} Q_{\alpha i}+H Z,
$$

with

$$
\begin{aligned}
\Omega^{a b} & =d \omega^{a b}+\omega^{a c} \wedge \omega^{c b}+\theta^{a} \wedge \theta^{b}+\psi^{i} \wedge\left(C \sigma^{a b}\right) \psi_{i}, \\
T^{a} & =d \theta^{a}+\omega^{a c} \wedge \theta_{c}+\frac{1}{2} \psi^{i} \wedge C \gamma^{a} \psi_{i}, \\
\varrho^{\alpha i} & =d \psi^{\alpha i}+\frac{1}{2} \omega^{b c}\left(\sigma_{b c} \psi\right)^{\alpha i}+\frac{1}{2} \theta \wedge \gamma \psi^{\alpha i}+\varepsilon^{i j} A \wedge \psi^{\alpha j}, \\
H & =d A+\frac{1}{2} \psi^{i} \wedge C \psi^{j} \varepsilon_{i j} .
\end{aligned}
$$

We substitute (3.10a) and (3.10b) into (3.6), but then (3.6) is no longer invariant under $\operatorname{SL}(2, C)$ gauge transformations, so this may be corrected by adding

$$
-\frac{1}{2}\left[\theta^{a} \wedge \theta^{b}+\psi^{i} \wedge\left(C \sigma^{a b}\right) \psi_{i}\right] \wedge \theta^{c} \wedge \theta^{d} \varepsilon_{a b c d},
$$

which will not affect the variation (3.1). Then the $L_{N=2}$ which corresponds to

$$
\begin{aligned}
\mathrm{OSP}(4 / 2) \text { is }= & \theta^{a} \wedge \theta^{b} \wedge\left(\Omega_{4}^{c d}-\frac{1}{3} f_{m n} f^{m n} \theta^{c} \wedge \theta^{d}+\psi^{i} \wedge C \sigma^{c d} \psi_{i}+\theta^{c} \wedge \theta^{d}\right) \varepsilon_{a b c d} \\
& -\frac{1}{2} \theta^{a} \wedge \theta^{b} \wedge\left(\theta^{c} \wedge \theta^{d}+\psi^{i} \wedge C \sigma^{c d} \psi_{i}\right) \varepsilon_{a b c d} \\
& -4 \psi^{i} \wedge C \gamma_{5} \gamma_{b} \varrho_{i} \wedge \theta^{b} \\
& -\frac{\alpha}{4}\left(4 d A+\psi^{i} \wedge C \psi^{j} \varepsilon_{i j}\right) \wedge \psi^{k} \wedge C \gamma_{5} \psi^{\ell} \varepsilon_{k \ell} \\
& -\alpha D f^{c d} \wedge \theta^{a} \wedge \theta^{b} \wedge A \varepsilon_{a b c d} \\
& +\alpha f^{c d} \wedge \psi^{i} \wedge C \gamma^{a} \psi_{i} \wedge \theta^{b} \wedge A \varepsilon_{a b c d} \\
& +\frac{\alpha}{2} f^{c d} \psi^{i} \wedge C \psi^{j} \wedge \theta^{a} \wedge \theta^{b} \varepsilon_{i j} \varepsilon_{a b c d}
\end{aligned}
$$

This is the unconcentrated $L_{N=2}$ obtained in $[6,4]$ (after redefining our $\psi$ in (3.11) $\rightarrow i \psi$ and dropping the $i$ factor in the final result of [4]). 
Finally and for later use, we exhibit the equations of motion following from Eq. (3.8):

Torsion Equation (variation of $\omega^{a b}$ ).

$$
2 \varepsilon_{a b c d} T^{c} \wedge \theta^{d}-2 \alpha f_{[a}^{m} \varepsilon_{b] m c d} \theta^{c} \wedge \theta^{d} \wedge A=0,
$$

from which we deduce that

$$
T^{a}=-\alpha f^{a b} \wedge A \wedge \theta_{b} .
$$

Matter Field Equation (variation of $f^{a b}$ ).

$$
2 \alpha \varepsilon_{a b c d} T^{c} \wedge \theta^{d} \wedge A+\alpha \varepsilon_{a b c d} \theta^{c} \wedge \theta^{d} \wedge H-\frac{2}{3} f_{a b} \varepsilon_{m n p q} \theta^{m} \wedge \theta^{n} \wedge \theta^{p} \wedge \theta^{q}==0
$$

from which we obtain

$$
H=\frac{4}{\alpha} f_{a b} \theta^{a} \theta^{b}
$$

Taking the pull back of (3.15) and using Eq. (2.8d), we have

$$
\frac{1}{2} e_{\mu}^{m} e_{v}^{n} F_{m n} d x^{\mu} \wedge d x^{\nu}=\frac{4}{\alpha} f_{m n} e_{\mu}^{m} e_{v}^{\mu} d x^{\mu} \wedge d x^{\nu}
$$

from which we obtain $F_{m n}=\frac{8}{\alpha} f_{m n}$. Using Eq. (2.17) we deduce that $\alpha=4$, which is the usual value for the $N=2$ supergravity coupling constant.

Gravitino Equation (variation of $\left.\bar{\psi}^{\alpha i}\right)\left(\psi^{\alpha i} C_{\alpha \beta}=\bar{\psi}_{\beta}^{i}\right)$.

$$
\begin{aligned}
& -8 \gamma_{5} \gamma_{a} Q^{\alpha i} \wedge \theta^{a}-8 \gamma_{5} \varepsilon^{i j} \psi_{j}^{\alpha} \wedge H+2 \alpha f^{a b} \gamma^{c} \psi^{\alpha i} \wedge \theta^{d} \wedge A \varepsilon_{a b c d} \\
& +\alpha f^{a b} \theta^{c} \wedge \theta^{d} \wedge \psi_{j}^{\alpha} \varepsilon_{a b c d} \varepsilon^{i j}+4 \gamma_{5} \gamma_{a} \psi^{\alpha i} \wedge T^{a}=0
\end{aligned}
$$

by substituting Eqs. (3.13) and (3.15) we get:

$$
\varrho^{\alpha i}=\varrho_{a b}^{\alpha i} \theta^{a} \wedge \theta^{b}+K \varepsilon^{i j}\left(\gamma_{a} \psi^{\alpha j} \wedge \theta_{b} f^{a b}+\frac{1}{2} \gamma_{5} \gamma_{a} \psi^{\alpha j} \wedge \theta_{b} f_{c d} \varepsilon^{a b c d}\right)
$$

where $K$ is equal to $\frac{\alpha}{4}$, and $\varrho_{a b}^{\alpha i}$ are the inner components which satisfies the RaritaSchwinger propagating equation for the spin 3/2-particles, that is

$$
\gamma_{5} \gamma_{a} \varrho_{b d}^{\alpha i} \varepsilon^{a b d e}=0
$$

Note that the above equation is equivalent to saying that the quantity $\varrho_{\text {Hor }}^{\alpha i}=\varrho_{a b}^{\alpha i} \theta^{a} \wedge \theta^{b}$ is a horizontal vector field in the bundle $E$.

Maxwell's Equation (variation of $A$ ).

$$
\begin{aligned}
& 4\left(\varrho^{i} \wedge C \gamma_{5} \psi^{i}-\psi^{i} \wedge C \gamma_{5} \varrho^{j}\right) \varepsilon_{i j}-\alpha D f^{a b} \theta^{c} \wedge \theta^{d} \varepsilon_{a b c d} \\
& \quad+\alpha f^{a b} \psi^{i} \wedge C \gamma^{c} \psi_{i} \wedge \theta^{d} \varepsilon_{a b c d}=0,
\end{aligned}
$$

by substituting Eqs. (3.13), (3.15), and (3.17) in (3.19) one gets :

$$
D f^{a b}=-\frac{2}{\alpha} \varepsilon^{a b c d} \psi^{\alpha i}\left(C \gamma_{5}\right)_{\alpha \beta} \varrho_{c d}^{\beta j} \varepsilon_{i j}
$$




\section{Invariances of the Lagrangian}

Evaluating the supersymmetry transformations and showing the invariance of (3.8) is most easily done when we reduce the bundle $(E, \pi, M)$ to one whose group is $\mathrm{SL}(2, C)[1]$.

Let $(E / \operatorname{SL}(2, C), j, M)$ be the associated bundle to $(E, \pi, M)$ with cross-section $\sigma$. Then we define the reduced bundle to be $\sigma^{-1} E=\{(x, \mu) ; \sigma(x)=\operatorname{pr}(u)\}$; $\sigma^{-1} E \subseteq M \times E$, pr: $E \rightarrow E / \mathrm{SL}(2, C)_{-1}, \mu=P_{2}(x, u), x=P_{1}(x, u), P_{2}: \sigma^{-1} E \rightarrow E$, and $P_{1}: \sigma^{-1} E \rightarrow M$. We are interested in bundles $\sigma^{-1} E$ upon which we can choose new torsion $T^{a}$ and $H$ such that $H=T^{a}=0$ on $\sigma^{-1} E$. In order to achieve this we have to construct $\sigma$ in such a way that the forms $\omega^{a b}$ and $S=\left(\theta^{a}, A\right)$ pulled back to $\sigma^{-1} E$ via $P_{2}$ are an absolute parallelism (in physical terms this means that the matrix $e_{\mu}^{a}$ has an inverse, and the central change acts trivially on the supersymmetric transformations induced on $\sigma^{-1} E$ ).

Now there is a natural group action on $M \times E$ by the extended super-Poincare group, namely $(x, u) \rightarrow(x, u g)$ and we consider the submanifolds $R_{g} \sigma^{-1} E$ defined by $(x, u) \in R_{g} \sigma^{-1} E$ if, and only if, $\sigma(x)=\operatorname{pr}\left(u g^{-1}\right)$. The above group action is then defined to be $R_{h}(x, v)=\left(x, v g^{-1} h g\right), h \in \mathrm{SL}(2, C)$. If $g \in \mathrm{SL}(2, C)$, then $R_{g} \sigma^{-1} E$ $=\sigma^{-1} E$, and any change in the forms is just a gauge transformation which is not of particular interest. Therefore we will assume that $g$ is not in the $\operatorname{SL}(2, C)$ subgroup and it is generated by an element of the superalgebra spanned by the $P_{a}$ and $Q_{\alpha i}$; in other words $g$ is a group element which is either a bosonic or fermionic translation.

Suppose now we choose $g$ such that $\omega^{a b}$ and $S$ still form an absolute parallelism on $R_{g} \sigma^{-1} E$ (we will assume that this has been done). Then we can find a horizontal one form $\psi^{a b}$ such that with respect to the connection $\omega^{\prime a b}=\omega^{a b}+\psi^{a b}$ the new torsion $T^{a}$ and $H$ are zero. A good choice of $g$ is $g=\exp t V$, with $V$ being a vertical vector field in $E$; this choice will allow us to find the infinitesimal form of the supersymmetry transformations such that these transformations do not mix with the usual gauge transformations induced by $\operatorname{SL}(2, C)$. These supersymmetric transformations are defined to be the pull back from $R_{g} \sigma^{-1} E$ of $\omega^{\prime}, S, \psi^{\alpha i}$ by $R_{g}$ to $\sigma^{-1} E$ with the above choice of $g$. By definition of $\omega^{a b}, f^{a b}$ and $A$ we have:

let

$$
\begin{gathered}
\omega(V)=f^{a b}(V)=A(V)=0 ; \\
\theta^{a}(V)=P^{a} \\
\psi^{\alpha i}(V)=\lambda^{\alpha i} .
\end{gathered}
$$

Then the infinitesimal change in $\theta^{a}, \psi^{\alpha i}, f^{a b}$, and $A$ will be given by Lie differentiation by $V\left(L_{V}\right)$. Using $L_{V}=d \circ \ell_{V}+\ell_{V} \circ d$ we have:

$$
\begin{aligned}
\delta \theta^{a} & =L_{V} \theta^{a}=d \theta^{a}(V)+\ell_{V}\left(T^{a}-\omega_{c}^{a} \wedge \theta^{c}-\frac{1}{2} \psi^{i} \wedge C \gamma^{a} \psi_{i}\right) \\
& =d P^{a}+\omega_{c}^{a} P^{c}-\lambda^{i} C \gamma^{a} \psi_{i} .
\end{aligned}
$$

Therefore

$$
\begin{gathered}
\delta \theta^{a}=D P^{a}-\lambda^{i} C \gamma^{a} \psi_{i}, \\
\delta A=L_{V} A=d A(V)+\ell_{V}\left(H-\frac{1}{2} \psi^{i} \wedge C \psi^{j} \varepsilon_{i j}\right)=-\lambda^{i} C \psi^{j} \varepsilon_{i j}, \\
\delta f^{a b}=L_{V} f^{a b}=d f^{a b}(V)+\ell_{V} d f^{a b}=\ell_{V}\left(D f^{a b}-[\omega, f]\right)=\ell_{V} D f^{a b} .
\end{gathered}
$$


Therefore

$$
\begin{gathered}
\delta f^{a b}=-\frac{2}{\alpha} \varepsilon^{a b c d} \lambda^{\alpha i}\left(C \gamma_{5}\right)_{\alpha \beta} \varrho^{\beta j} \varepsilon_{i j} \\
\delta \psi^{\alpha i}\left(\text { extended super-Poincaré) }=L_{v} \psi^{\alpha i}=d \psi^{\alpha i}(V)+\ell_{V}\left(\varrho^{\alpha i}-\frac{1}{2} \omega^{b c}\left(\sigma_{b c} \psi\right)^{\alpha i}\right)\right. \\
=d \lambda^{\alpha i}+\frac{1}{2} \omega^{b c}\left(\sigma_{b c} \lambda\right)^{\alpha i}+\ell_{V} \varrho^{\alpha i} \\
=D \lambda^{\alpha i}+K \varepsilon^{i j}\left(\gamma^{c} \lambda^{\alpha j} \theta^{b} f_{c b}+\frac{1}{2} \gamma_{5} \gamma^{c} \lambda^{\alpha j} \theta^{b} f^{m n} \varepsilon_{c b m n}\right. \\
-K \varepsilon^{i j}\left(\gamma^{c} \psi^{\alpha j} P^{b} f_{c b}+\frac{1}{2} \gamma_{5} \gamma^{c} \psi^{\alpha j} P^{b} f^{m n} \varepsilon_{c b m n}\right) \\
\delta \psi^{\alpha i}(\operatorname{OSP}(4 / 2))=D \lambda^{\alpha i}+K \varepsilon^{i j}\left(\gamma^{c} \lambda^{\alpha j} \theta^{b} f_{c b}+\frac{1}{2} \gamma_{5} \gamma^{c} \lambda^{\alpha j} \theta^{b} f_{m n} \varepsilon_{c b m n}\right) \\
+\frac{1}{2} \lambda^{\alpha i} \theta \gamma+\varepsilon^{i j} \lambda^{\alpha j} A \\
-\left(\frac{1}{2} \psi^{\alpha i} P \gamma+K \varepsilon^{i j}\left(\gamma^{c} \psi^{\alpha j} \theta^{b} f_{c b}+\frac{1}{2} \gamma_{5} \gamma^{c} \psi^{\alpha j} P^{b} f_{m n} \varepsilon_{c b m n}\right)\right.
\end{gathered}
$$

In the above derivation we have used Eqs. (2.6), (3.10b), (3.17), (3.20) and the fact that $\ell_{V} T^{a}=\ell_{V} H=\ell_{V} \varrho_{H o r}^{\alpha i}=0$ since $V$ is a vertical vector field in $E$. Equations (4.1), (4.4), and (4.5) are the generalized supersymmetry transformations of $L_{N=2}$ supergravity. This is an important difference between our work and that of $[3,4]$. Moreover the above construction of supersymmetry transformations was a direct consequence of bundle reduction, and thus it arises in a purely geometrical way. This is a considerable improvement on the method of [9]. In addition the above construction overcomes the interpretation of the "non-geometrical" terms which arise in [9] and their link to the supersymmetry transformations.

Most uses of the invariances (4.1), (4.4), and (4.5) have focussed on the case $P_{c}=0$, based on the assumption that a variation with respect to a non-zero $P_{c}$ corresponds to a gauge transformation, which is not the case when we compare (4.1)-(4.5) with the usual gauge transformations [1]. In fact from their construction, it is easy to see that neither $P_{c}=0$ nor $\lambda^{\alpha i}=0$ transformations can be thought of as gauge transformations. Therefore it is not surprising that the case $P_{c}=0$ has lead to many problems in quantization techniques of supergravity. These problems are mainly in using Fadeev-Popov ghosts which lead to the incorrect $S$ matrix of relativistic systems describing boson and fermions first and second class constraints [10]. So we expect $P_{c} \neq 0$ will have considerable importance in understanding quantum aspects of supergravity theories.

To show the invariance of (3.8) under transformations (4.1)-(4.4) we calculate $L_{V} L^{\prime}=\delta L$ where $L^{\prime}$ is the Lagrangian constructed using $\omega^{\prime a b}$ defined on $R_{g} \sigma^{-1} E$; then

$$
\delta L=L_{V} L^{\prime}=d_{\ell_{V}} L^{\prime}+\ell_{V} d L^{\prime}
$$

Consider now the bundle $(E, \operatorname{Pr}, E / \mathrm{SL} /(2 C))$. This bundle is a principal bundle with group $\operatorname{SL}(2, c)$ and connections $\omega$ over this bundle $L_{N=2}$ is, by construction, a horizontal four-form invariant by right multiplication of $\operatorname{SL}(2, c)$, and hence with $D$ being the horizontal exterior derivative we have $d L=D L[7]$. Therefore

$$
\delta L=d_{\ell_{V}} L^{\prime}+\ell_{V} D L^{\prime}
$$


From Eqs. (2.6) we have

$$
\begin{aligned}
\Omega_{E}^{a b} & =D \omega^{a b}, \\
\varrho^{\alpha i} & =D \psi^{\alpha i}, \\
T^{a} & =D \theta^{a}+\frac{1}{2} \psi^{i} \wedge C \gamma^{a} \psi_{i}, \\
H & =D A+\frac{1}{2} \psi^{i} \wedge C \psi^{j} \varepsilon_{i j},
\end{aligned}
$$

and from Bianchi's identity $D \Delta=0$ we have

$$
\begin{aligned}
D \Omega_{E}^{a b} & =0, \\
D T^{a} & =\Omega_{E}^{a c} \wedge \theta_{c}-\psi^{i} \wedge C \gamma^{a} \varrho_{i}, \\
D \varrho^{\alpha i} & =\frac{1}{2} \Omega_{E}^{b c}\left(\sigma_{b c} \psi\right)^{\alpha i}, \\
D H & =-\psi^{i} \wedge C \varrho^{j} \varepsilon_{i j} .
\end{aligned}
$$

Using Eqs. (4.8) and (4.9) we arrive at:

$$
D L^{\prime}=2 T^{a} \wedge\left(\theta^{b} \wedge \Omega_{E}^{c d} \varepsilon_{a b c d}+2 \psi^{i} \wedge C \gamma_{5} \gamma_{a} \varrho_{i}\right)+4 H \wedge\left(\psi^{i} \wedge C \gamma_{5} \varrho^{j}-\varrho^{i} \wedge C \gamma_{5} \psi^{j}\right) \varepsilon_{\imath j}
$$

However $T^{a}=H=0$ on $\sigma^{-1} E$, therefore $D L^{\prime}=0$ and we have $\delta L=d_{\ell_{V}} L^{\prime}$, i.e. a total divergence which can be ignored as a surface integral. Therefore the supersymmetry transformations are invariances of Eq. (3.8). The above analysis is well applicable to the gauged $N=2$ supergravity given by Eq. (3.11).

From the above construction presented in this section one can show that by the standard theorems, we can always construct a bundle $F$ reducible from $E$ such that in this bundle $\omega^{a b}$ and $\theta^{a}$ form an absolute parallelism and therefore we can always choose a new torsion $T^{a}$ such that $T^{a}=0$ in $F$. As a consequence a new $L_{N=2}$ of supergravity can be found which is invariant under the supersymmetric transformations (4.10)-(4.5) and under $O(2)$ gauge transformations. It is given by [using Eqs. (3.10) and (3.11)]

$$
\begin{aligned}
L_{N=2}= & \theta^{a} \wedge \theta^{b} \wedge\left(\Omega_{4}^{c d}-\frac{1}{3} f_{m n} f^{m n} \theta^{c} \wedge \theta^{d}+\theta^{c} \wedge \theta^{d}+\psi^{i} \wedge C \sigma^{c d} \psi_{i}\right) \varepsilon_{a b c d} \\
+ & \varepsilon_{a b c d}\left[-\frac{1}{2} \theta^{a} \wedge \theta^{b} \wedge\left(\theta^{c} \wedge \theta^{d}+\psi^{i} \wedge C \sigma^{c d} \psi_{i}\right)\right]-4 \psi^{i} \wedge C \gamma_{5} \gamma_{a} \varrho_{i} \wedge \theta^{a} \\
& -4 H \wedge \psi^{k} \wedge C \gamma_{5} \psi^{\ell} \varepsilon_{k \ell}+2 \psi_{i} \wedge C \psi_{j} \wedge \psi^{i} \wedge C \gamma_{5} \psi^{j} \\
& +\alpha f^{a b} \theta^{c} \wedge \theta^{d} \wedge H \varepsilon_{a b c d} .
\end{aligned}
$$

\section{Conclusion}

Unlike other geometrical approaches to supergravity, by introducing a central charge, we have obtained $N=2$ supergravity in a simple, natural geometrical way, with the symmetry group the super-Poincare group. Then we have shown that by shifting the charge from being central we could obtain the extension of $L_{N=2}$ (super-Poincaré) which is $L_{N=2}(\mathrm{OSP}(4 / 2))$. As a consequence of our construction we could show that the supersymmetry transformations can be generalized nontrivially in such a way that they do not mix with the usual gauge transformations. Another interesting result is the fact that the coupling constant of $N=2$ supergravity has been determined by requiring $Z$ invariance rather than $P_{a}$ and $Q_{\alpha i}$ 
invariances which is the case of $[5,6]^{2}$. Moreover it is shown that the invariance of the lagrangian (3.8) can be shown in a straightforward manner when we reduce our bundle to one whose group is $\operatorname{SL}(2, c)$.

We expect that due to the success of this simple approach to $N=2$ supergravity, the derivation of $N=8$ supergravity and its generalized supersymmetric transformations will be relatively straightforward. We will leave for further publication a fibre bundle treatment of off-shell versions of $N=1$ and $N=2$ supergravity.

Acknowledgements. I am extremely grateful to Prof. C. J. Isham for his guidance and help, Dr. A. Rogers for her collaboration, contribution and useful suggestions, M. J. Duff for his support and encouragement, C. N. Pope for useful conversations, and K. Stelle for pointing out useful remarks.

\section{References}

1. Yates, R.G.: Fibre bundles and supersymmetries. Commun. Math. Phys. 76, 255-268 (1980)

2. Chamseddine, A.H., West, P.C.: Supergravity as a gauge theory of supersymmetry. Nucl. Phys. B129, 39-44 (1977)

MacDowell, S.W., Mansouri, F.: Unified geometric theory of gravity and supergravity. Phys. Rev. Lett. 38, 739-741 (1977)

3. Fre, P.: Group manifold first-order formulation of $N=2, d=4$ supergravity theory. Nucl. Phys. B179, 417-440 (1981)

4. D'Auria, R., Fre, P., Regge, T.: Graded-Lie algebra cohomology and supergravity. Riv. Nuovo Cimento 3, 12 (1980)

5. Ferrara, S., van Nieuwenhuizen, P.: Consistent supergravity with complex spin $-\frac{3}{2}$ gauge fields. Phys. Rev. Lett. 37, 1669 (1976)

6. Freedman, D.Z., Das, A.: Gauge internal symmetry in extended supergravity. Nucl. Phys. B120, 221 (1976)

7. Kobayashi, S., Nomizu, K.: Foundations of differential geometry, Vols. I and II. New York, London: Interscience (1963)

8. Cho, Y.M.: Higher-dimensional unifications of gravitation and gauge theories. J. Math. Phys. 16, 2029 (1975)

Bleecker, D.: Gauge theory and variational principles. Reading, MA: Addison-Wesley, Series A 1981

9. Townsend, P.K., van Nieuwenhuizen, P.: Geometrical interpretation of extended supergravity. Phys. Lett. 67B, 439 (1977)

10. Fradkin, E.S., Fradkina, T.E.: Quantization of relativistic systems with boson and fermion firstand second-class constraints. Phys. Lett. 72B, 343 (1978)

Communicated by S. Hawking

Received February 2, 1983; in revised form March 24, 1983

Note added in proof. The invariance of the Lagrangian (3.8) can also be shown using a simpler method than that which is outlined in Sect. IV. By contracting equation (4.11) invariance may be shown on $M_{4}$ rather than $\sigma^{-1} E$. From (4.7) we have $\delta L_{M}=d_{\ell_{V}} L_{M}^{\prime}+/_{V} D L_{M}^{\prime}$. Using the Bianchi identities in (4.9) we have

$$
\begin{aligned}
D L_{M}^{\prime}= & 2 T^{a} \wedge\left(\theta^{b} \wedge \Omega_{E}^{c d} \varepsilon_{a b c d}+2 \psi^{i} \wedge C \gamma_{5} \gamma_{a} \varrho_{i}+\alpha \theta^{b} \wedge f^{c d} \wedge H \varepsilon_{a b c d}\right) \\
& +8 H \wedge \psi^{l} \wedge C \gamma_{5} \varrho^{j} \varepsilon_{\imath j}-4 \varrho^{i} \wedge C \gamma_{5} \gamma_{a} \varrho_{l} \wedge \theta^{a}+\alpha D f^{a b} \wedge \theta^{c} \wedge \theta^{d} \wedge H \varepsilon_{a b c d} \\
& -\alpha f^{a b} \psi^{l} \wedge C \gamma^{c} \psi_{\imath} \wedge \theta^{d} \wedge H \varepsilon_{a b c d}-\alpha f^{a b} \wedge \theta^{c} \wedge \theta^{d} \wedge \psi^{i} \wedge C Q^{j} \varepsilon_{t j} \varepsilon_{a b c d} .
\end{aligned}
$$

2 Kellogg S. Stelle (private communication) 
Set $P^{a}=0$ on $M_{4}$ and choose $\omega^{a b}$ such that $T^{a}=0$. Use of equations (3.17) and (3.20) leads to $D L_{M}^{\prime}=-4 \varrho_{\text {Hor }}^{i} \wedge C \gamma_{5} \gamma_{a} \varrho_{i \text { Hor }} \wedge \theta^{a}$. This implies that $\ell_{V} D L_{M=0}^{\prime}$ since $\ell_{V} \varrho_{\text {Hor }=0}^{i}$ and $\ell_{V} \theta^{a}=\theta^{a}(v)=P^{a}=0$. Hence $\delta L_{M}=d_{\ell_{V}} L_{M}^{\prime}$ is a total divergence which can be ignored. The main idea behind this procedure is that the variation of the supergravity Lagrangian under super symmetry transformations is equivalent to the substitution of (3.17) and (3.20) which are responsible for deriving the supersymmetry transformations into $\delta L_{M}$. 